\title{
Dissolution study and thermal analysis of fenofibrate-Pluronic F127 solid dispersions
}

\author{
Bożena Karolewicz ${ }^{1}$ Maciej Gajda ${ }^{1} \cdot$ Janusz Pluta $^{1} \cdot$ Agata Górniak $^{2}$
}

Received: 2 July 2015/Accepted: 29 August 2015/Published online: 14 September 2015

(C) The Author(s) 2015. This article is published with open access at Springerlink.com

\begin{abstract}
Prepared solid dispersions were applied to a drug with low water solubility to improve its dissolution rate. Fenofibrate (FEN) is a Biopharmaceutical Classification System (BCS) class-II (poorly water-soluble) drug, and its bioavailability is limited by the dissolution rate. The physical state of FEN in solid dispersions with Pluronic F127 (PLU) prepared using the fusion method was assessed using Fourier transform infrared spectroscopy (FTIR), X-ray powder diffraction (XRPD), and differential scanning calorimetry (DSC). The DSC investigation revealed that FEN and PLU form a simple eutectic system. The DSC, XRPD, and FTIR studies of the investigated solid dispersions revealed no interaction between FEN and PLU. The intrinsic dissolution rate (IDR) of FEN from solid dispersions was significantly increased compared with the pure drug. The enhancement in the intrinsic dissolution rate was approximately 134 -fold for solid dispersions containing 30/70 \% w/w of FEN/PLU.
\end{abstract}

Keywords Fenofibrate Pluronic F127 · Solid dispersion · DSC studies · Intrinsic dissolution rate $\cdot$ XRPD

Maciej Gajda

maciej.gajda@umed.wroc.pl

1 Department of Drug Form Technology, Wroclaw Medical University, Borowska 211A, 50-556 Wrocław, Poland

2 Laboratory of Elemental Analysis and Structural Research, Wroclaw Medical University, Borowska 211A, 50-556 Wrocław, Poland

\section{Introduction}

Fenofibrate (FEN) (isopropyl ester of 2-[4-(4-chloro-benzoyl) phenoxy]-2-methylpropanoic acid) is a Biopharmaceutical Classification System (BCS) class-II, lowsolubility, high-permeability hypolipidemic drug [1]. It is used to treat hypercholesterolemia, heart disease, and diabetic complications [2]. The compound is practically insoluble in water $\left(0.1 \mu \mathrm{g} \mathrm{m}^{-1}\right)$, which limits its absorption from the gastrointestinal tract [3]. Several methods have been employed to improve the solubility of FEN, including micronization [4-6], preparation of solid dispersions [7, 8], preparation of eutectic mixtures with polymers [3], formulation of nanosuspension [5], self-microemulsifying drug delivery systems [9], and an inclusion complex with $\beta$-cyclodextrin [10], the addition of surfactants, such as sodium lauryl sulphate, [11], the formulation of the drug into granules using slugging and liquisolid compaction techniques [12], or the melt granulation technique [13, 14].

The FEN solid dispersions in Eudragit ${ }^{\circledR}$ E100 and polyvinylpyrrolidone-vinyl acetate copolymer S630 (PVPVA) were prepared using a hot-melt extrusion method, which improved the dissolution and thus the bioavailability of the drug [4]. The solid dispersion technique is successfully used to improve the dissolution rate and bioavailability of FEN $[15,16]$. Tablets formulated with a solid dispersion of FEN with hydroxy propyl- $\beta$-cyclodextrin produced an enhanced drug dissolution rate. The in vivo studies of rats showed a significant decrease in cholesterol, triglycerides, low-density lipoprotein (LDL), and very lowdensity lipoprotein (VLDL) levels and an increase in highdensity lipoprotein (HDL) levels compared with the pure drug-treated group [16].

Pluronics are nonionic surfactants composed of polyoxyethylene-polyoxypropylene-polyoxyethylene triblock 
copolymers with a melting temperature of $52-57{ }^{\circ} \mathrm{C}$. The observed increased drug dissolution rate in solid dispersions with the polymer is likely due to the decreased particle size, reduced drug crystallinity, and surface active properties of pluronic itself. The solid dispersion of FEN in Poloxamer 188 and tocopheryl polyethylene glycol succinate as a surfactant prepared using spray drying significantly increased the dissolution rate of this drug [7]. Tejas et al. obtained solid dispersions of FEN using PEG 6000, Poloxamer 407, and a mixture of PEG 6000 and Poloxamer 407 (1:1 mixture) using solvent, fusion, and lyophilization methods. The lyophilized Poloxamer 407 solid dispersions had a greater effect on the rate and extent of the FEN dissolution [17]. The enhanced FEN dissolution from lyophilized dispersions explained the decreased drug crystallinity in these formulations [18]. In addition, improvements in the FEN dissolution property were observed for solid dispersions of the drug with Poloxamer 407 and Poloxamer 407 microprepared using a physical mixing technique [19]. Irwan et al. [20] prepared the FEN solid dispersions with Poloxamer 407 using a solvent evaporation method and observed a significantly faster release of the drug when the fenofibrate loading was not greater than $50 \%$.

In the present work, the FEN solid dispersions with PLU in mass ratios of 10/90, 20/80, 30/70, 40/60, 50/50, 60/40, 70/30, $80 / 20$, and $90 / 10$ were prepared using the fusion method. Differential scanning calorimetry (DSC), X-ray powder diffraction (XRPD), and Fourier transform infrared spectroscopy (FTIR) were used to investigate the properties of the obtained solid dispersions of FEN-PLU series. Intrinsic dissolution studies of the obtained formulations were performed in $0.5 \% \mathrm{SLS}$ w/v and were compared with pure FEN.

\section{Experimental}

\section{Materials}

FEN (99 \% purity) and PLU were supplied by SigmaAldrich (USA). Sodium lauryl sulphate (SLS) was purchased from Stanlab (Poland). Acetonitrile HPLC grade was obtained from JT Baker (The Netherlands). Phosphoric acid $(85 \%)$ was obtained from Sigma (Germany).

\section{Preparation of solid dispersions}

Accurately weighed amounts of PLU were mixed with an appropriate amount of FEN in a porcelain dish and heated on an electrical plate to $80-85{ }^{\circ} \mathrm{C}$ with continuous stirring to obtain homogeneous preparations, which were then rapidly cooled over an ice bath. The total mass of each solid dispersion was $5.0 \mathrm{~g}$. The obtained solid dispersions were stored in a desiccator under vacuum for $24 \mathrm{~h}$ and then pulverized using an agate mortar and pestle. The pulverized powders were sieved using a $315-\mu \mathrm{m}$ sieve and then stored in a desiccator at room temperature until use. The mass ratios of the mixtures were 90/10, 80/20, 70/30, 60/40, 50/50, 40/60, $30 / 70,20 / 80$, and 10/90 \% w/w, respectively.

\section{Drug content}

The equivalent mass of solid dispersions containing $10 \mathrm{mg}$ of FEN were accurately weighed and dissolved in $100 \mathrm{~mL}$ of acetonitrile. The solution was filtered, and the FEN content was analysed using HPLC.

\section{Differential scanning calorimetry (DSC)}

The DSC curves of each mixture were obtained using Mettler Toledo DSC 25 equipped with a heat flow sensor and interfaced with a computer using an interface TA Controller TC 15. Measurements were performed using the $\mathrm{STAR}^{\mathrm{e}}$ software. The samples for DSC measurements were sealed in $40-\mu \mathrm{L}$ standard aluminium crucibles with a single hole punched in the lid. The total mass of each sample was between 5 and $7 \mathrm{mg}$. An empty pan of the same type was employed as a reference. The DSC instrument was calibrated using the melting point of indium $\left(156.6 \pm 0.3{ }^{\circ} \mathrm{C}\right)$ as a standard. The DSC scans of each mixture were performed at a heating rate of $5{ }^{\circ} \mathrm{C} \mathrm{min}^{-1}$ in the temperature range from 25 to $200{ }^{\circ} \mathrm{C}$. The DSC cell was purged with a stream of dry argon at a flow rate of $50 \mathrm{~mL} \mathrm{~min}{ }^{-1}$. The experiments were performed in triplicate, and the mean values were calculated.

\section{X-ray powder diffraction analysis (XRPD)}

The XRPD patterns were recorded using a powder diffractometer (D2 Phaser, Brüker) with $\mathrm{CuK} \alpha$ radiation with a LYNXEYE detector. The degree of diffractions was measured at $15^{\circ} \mathrm{min}^{-1}$ from $5^{\circ}$ to $60^{\circ}(2 \theta)$ with an accuracy of $0.02^{\circ}$ throughout the measurement range at $0.5 \mathrm{~s} \mathrm{step}^{-1}$.

\section{Fourier transform infrared spectroscopy (FTIR)}

FTIR spectra were measured using a Spectrum Two spectrophotometer (PerkinElmer). Spectra were recorded in the $450-4000 \mathrm{~cm}^{-1}$ wavenumber range with a resolution of $4 \mathrm{~cm}^{-1}$, averaged over 32 scans.

\section{Intrinsic dissolution rate (IDR) studies}

IDR studies were conducted using a rotating-disc system under sink conditions in $1000 \mathrm{~mL}$ of distilled water containing $0.5 \% \mathrm{w} / \mathrm{v}$ SLS at $37 \pm 0.5{ }^{\circ} \mathrm{C}$ and rotational 
Table 1 Drug content in the prepared solid dispersions

\begin{tabular}{lc}
\hline Formulation code & Average content of FEN \\
\hline 10/90 FEN/PLU & $98.51 \pm 0.25$ \\
20/80 FEN/PLU & $97.55 \pm 0.12$ \\
30/70 FEN/PLU & $97.56 \pm 0.24$ \\
40/60 FEN/PLU & $98.55 \pm 0.35$ \\
50/50 FEN/PLU & $98.91 \pm 0.15$ \\
60/40 FEN/PLU & $98.28 \pm 0.32$ \\
70/30 FEN/PLU & $99.57 \pm 0.14$ \\
80/20 FEN/PLU & $97.53 \pm 0.19$ \\
90/10 FEN/PLU & $100.98 \pm 0.17$ \\
\hline
\end{tabular}

Data are expressed as a mean $\pm \mathrm{SD}(n=3)$

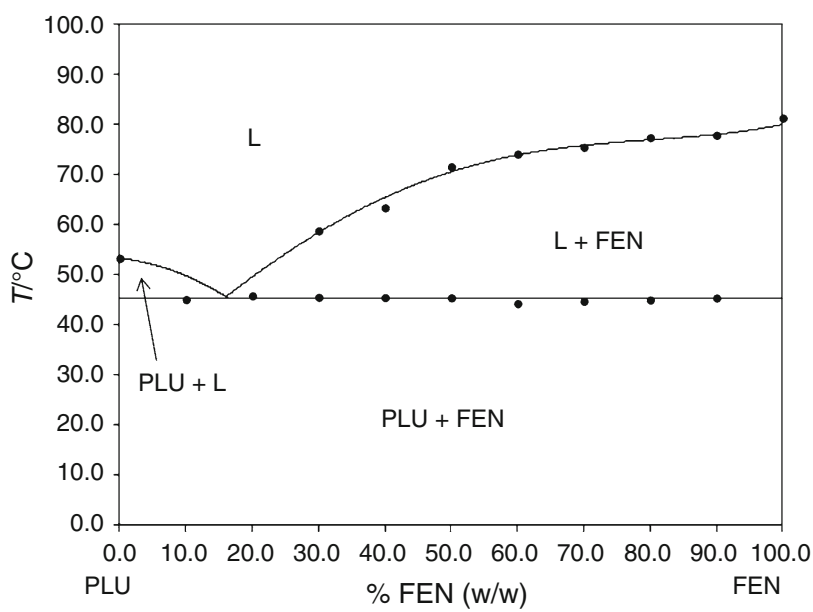

Fig. 1 Phase diagram of the FEN-PLU system

speeds of $50 \mathrm{rpm}$. The dissolution system was fitted with SR8-PLUS (Hanson) and a six-channel peristaltic pump. FEN (100.0 mg) or an equivalent amount of solid dispersion discs were prepared by compressing the powder in a hydraulic press (Specac, Mettler Toledo) for 2 min under $1 \mathrm{t}$ of compression force using an 8-mm punch. The samples were withdrawn at appropriate time intervals, namely, $5,10,15,20,30,40,50,60,90$ and $120 \mathrm{~min}$. Additional trials were performed at sampling times of $240,480,360$, 720,1080 , and 1440 min to monitor the dissolution behaviour of the drug over a 24 -h time period. The quantitative measurements of FEN were performed using an HPLC system (System Agilent 1260 Infinity Series) with an eightchannel DAD detector. The analysis was accomplished using Zorbax SB-C18 (Agilent) $150 \times 4.6 \mathrm{~mm}, 5 \mu \mathrm{m}$. The analysis was performed using an isocratic elution with a mixture of solvents on the composition of $70 \% \mathrm{v} / \mathrm{v}$ acetonitrile and $30 \% \mathrm{v} / \mathrm{v}$ water that was adjusted to $\mathrm{pH} 2.5$ by adding phosphoric acid with a steady flow rate of $2.5 \mathrm{~mL} \mathrm{~min}^{-1}$ (volume injections of $20 \mu \mathrm{L}$ ). Substances eluted from the column were identified using a UV visible detector at $286 \mathrm{~nm}$. External FEN standards were used to obtain the calibration curves. Evaluation methods were linear in the range of $0.5-12.0 \mu \mathrm{g} \mathrm{mL}^{-1}$ for both compounds (linearity $r^{2}=0.9999$ ).

\section{Results and discussion}

\section{Drug content}

The FEN content in solid dispersions was found to be in range of $97.55-100.98 \%$ of the declared amount (Table 1).

\section{DSC study}

The DSC curves of pure FEN and PLU indicate only one sharp endothermal effect $\left(\Delta_{\text {fus }} H=79.3\right.$ and $86.3 \mathrm{~kJ} \mathrm{~mol}^{-1}$, respectively) that corresponds to the melting points at 81.4 and $53.4{ }^{\circ} \mathrm{C}$, respectively. This confirms that the substances are stable and do not decompose under the experimental conditions. The phase diagram of the investigated system constructed based on the DSC results is shown in Fig. 1.

The DSC curves of the pure components and obtained dispersions are given in Fig. 2. The DSC curves of the mixtures indicate that FEN and PLU formed a simple binary eutectic system - only two kinds of thermal effects are shown for the entire range of compositions. The onset of the first peak consistently appeared near $45.3{ }^{\circ} \mathrm{C}$ (Fig. 2), which indicated the following eutectic reaction:

\section{Solid fenofibrate (FEN) + solid Pluronic F127 (PLU)} $=$ liquid $(\mathrm{L})$.

The second peak, which corresponds to the temperature of liquid, was generally wider. This indicated complete melting over a temperature range. In this two-phase region (Fig. 1, L + FEN, PLU + L), the peak broadening was the result of the solid phase content (FEN or PLU). Terminal solid solutions were not observed on either side of the phase diagram. The values of eutectic melting enthalpy $\Delta H$ for a given mixture, determined by integrating the eutectic peak area of the DSC curves, are plotted in Fig. 3 versus a mass fraction of FEN (Tamman's triangle construction [21]). The thermal effect of the eutectic transition goes to zero for a composition that corresponds to pure FEN, confirming that there is no mutual miscibility in the solid state and no formation of a terminal solid solution. The values of eutectic melting enthalpy $\Delta H$ (Fig. 3, filled triangles) increase linearly with the PLU content. Near the eutectic point, the DSC scans showed a characteristic overlap of two endotherms (eutectic and liquid events) into a single peak. Thus, the eutectic composition was determined by plotting the non-eutectic melting enthalpy (after 


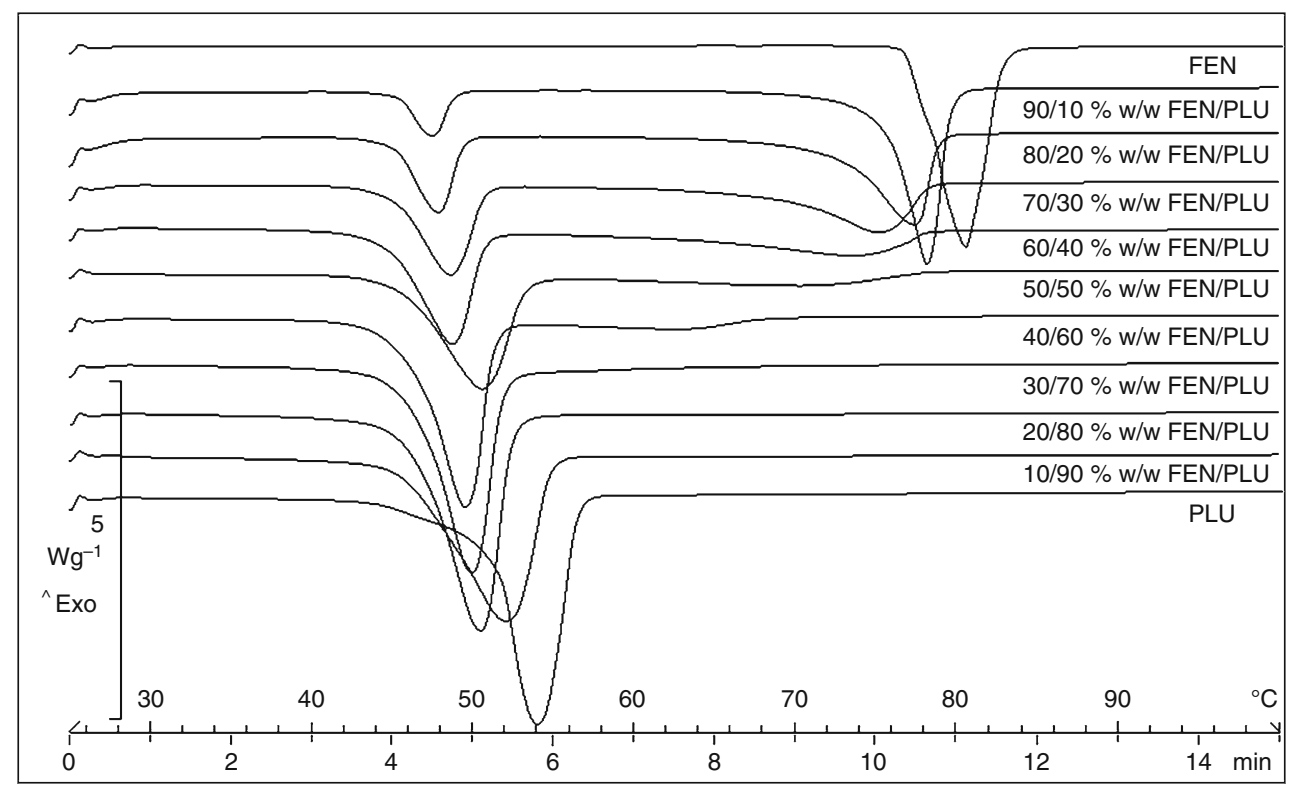

Fig. 2 DSC curves of pure FEN, PLU, and the obtained FEN/PLU solid dispersions

eutectic melting) of FEN as a function of the mass percentage of the drug and extrapolating the fitted line to zero enthalpy (Fig. 3, filled circles). Finally, the parameters of the eutectic point $(\mathrm{E})$ have been established as follows:

1. Eutectic composition: mass fraction of FEN $16.5 \%$, mass fraction of PLU $83.5 \%$;

2. Eutectic temperature: $45.3{ }^{\circ} \mathrm{C}$.

\section{XRPD study}

The XRPD patterns of FEN, PLU, and solid dispersions are presented in Fig. 4. As a crystalline compound, FEN

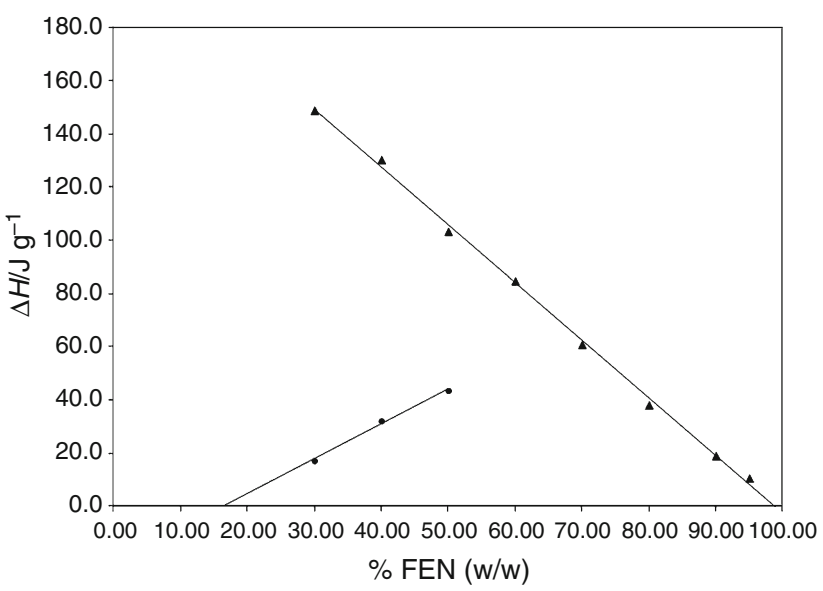

Fig. 3 Eutectic melting enthalpy $\Delta H$ at $45.3{ }^{\circ} \mathrm{C}$ (filled triangles) and the non-eutectic melting enthalpy (filled circles) versus the FEN mass ratio

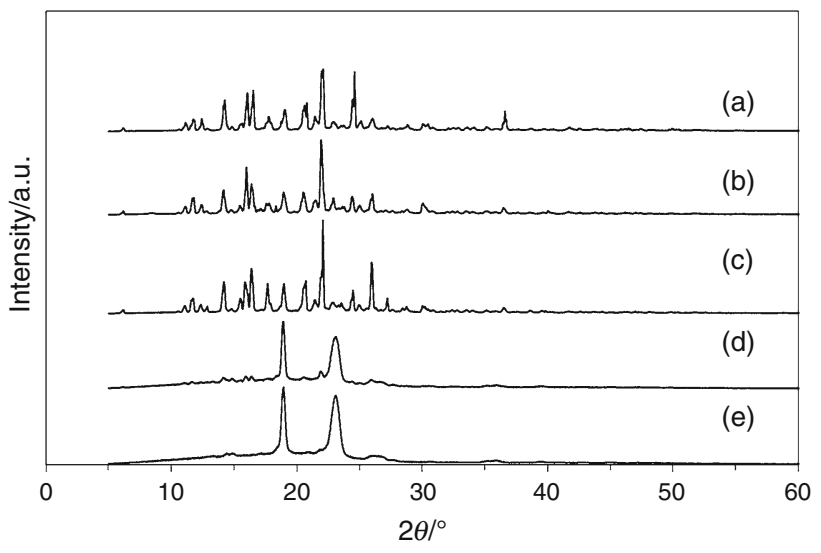

Fig. 4 XRPD patterns of FEN (a), 95/5\% w/w FEN/PLU (b), 90/10 \% w/w FEN/PLU $(c), 10 / 90 \%$ w/w FEN/PLU $(d)$ and PLU $(e)$

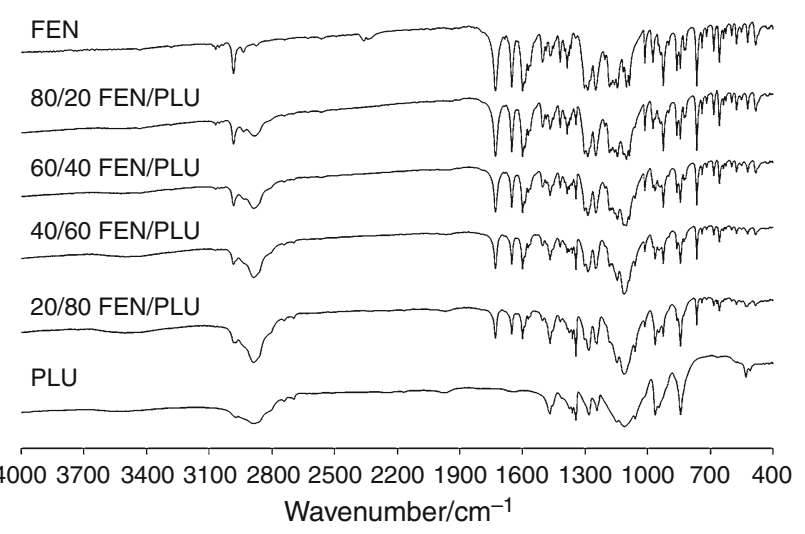

Fig. 5 FTIR spectra of PLU, FEN, and its solid dispersions 


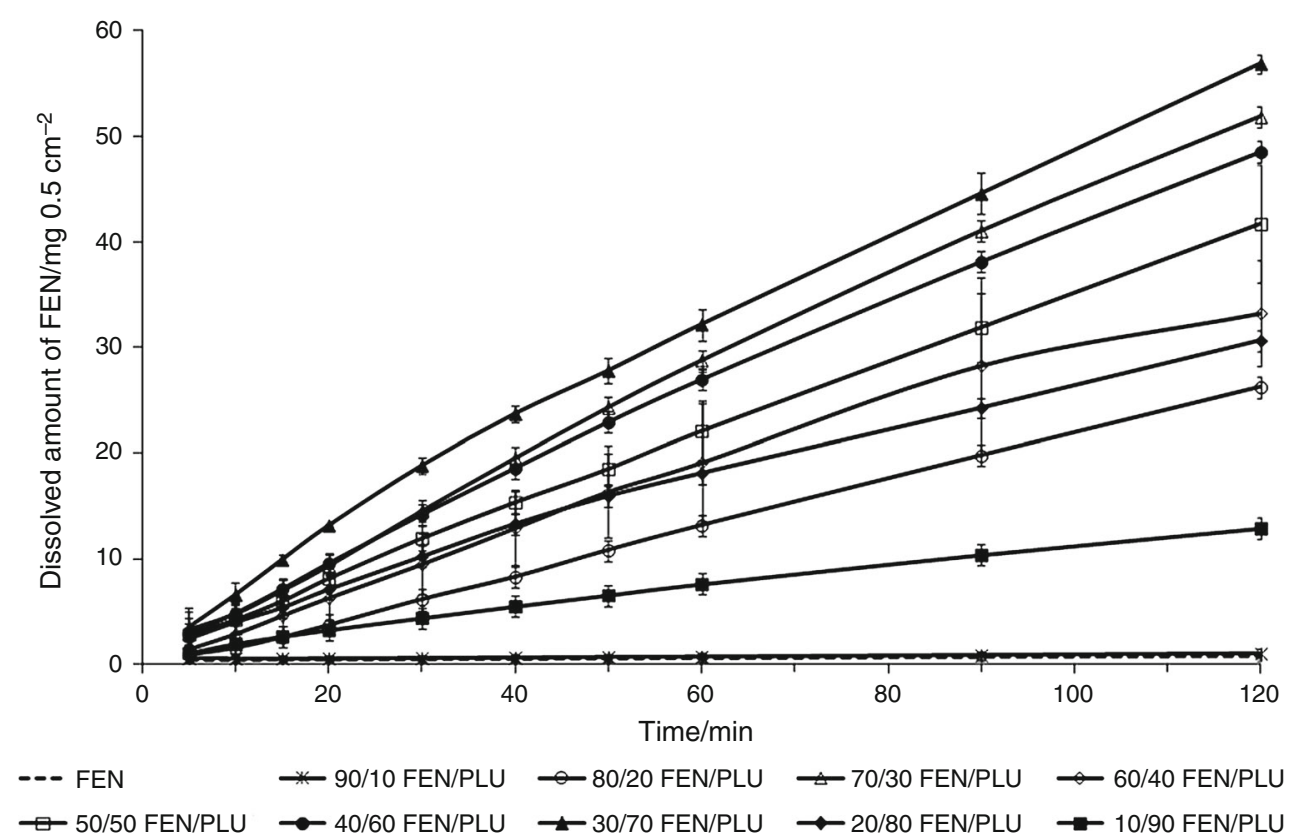

Fig. 6 Dissolved amount of pure FEN and its solid dispersions with PLU

Table 2 IDR of pure FEN and the prepared solid dispersions and the corresponding ratios

\begin{tabular}{llll}
\hline Formulation code & IDR/mg cm ${ }^{-2} \mathrm{~min}^{-1}$ & $R^{2}$ & IDR ratio SDs/FEN $^{\mathrm{a}}$ \\
\hline 10/90 FEN/PLU & $0.102 \pm 0.002$ & 0.9928 & 27.99 \\
20/80 FEN/PLU & $0.246 \pm 0.026$ & 0.9894 & 72.08 \\
30/70 FEN/PLU & $0.459 \pm 0.004$ & 0.9926 & 134.38 \\
40/60 FEN/PLU & $0.402 \pm 0.035$ & 0.9964 & 117.76 \\
50/50 FEN/PLU & $0.340 \pm 0.033$ & 0.9992 & 99.60 \\
60/40 FEN/PLU & $0.289 \pm 0.052$ & 0.9893 & 84.58 \\
70/30 FEN/PLU & $0.435 \pm 0.064$ & 0.9962 & 127.34 \\
80/20 FEN/PLU & $0.225 \pm 0.041$ & 0.9994 & 65.84 \\
90/10 FEN/PLU & $0.004 \pm 0.001$ & 0.9541 & 1.17 \\
FEN & $0.003 \pm 0.000$ & 0.9976 & -
\end{tabular}

Data are expressed as a mean $\pm \mathrm{SD}(n=3)$

${ }^{a}$ SDs-solid dispersions

showed a series of peaks at $11.77^{\circ}, 14.24^{\circ}, 16.06^{\circ}, 16,53^{\circ}$, $20.62^{\circ}, 21.97^{\circ}, 24.62^{\circ}$ and $36.58^{\circ}$. The X-ray diffractograms of pure PLU showed distinct peaks at $18.93^{\circ}$ and $23.10^{\circ}$. The diffraction patterns of FEN in solid dispersions were similar to those of the pure drug, suggesting a simple mixing of drugs and carriers.

\section{FTIR study}

FTIR spectra of FEN, PLU and 80/20, 60/40, 40/60, and 20/80 \% w/w FEN/PLU, respectively, are shown in Fig. 5. The pure drug spectra show characteristic peaks at 2983 and $2935 \mathrm{~cm}^{-1}$ due to the benzene ring, at $1729 \mathrm{~cm}^{-1}$ due to the ester group and at 1651 and $1599 \mathrm{~cm}^{-1}$ due to the lactone carbonyl functional group. The PLU FTIR spectrum is characterized by principal absorption peaks at 2889 (C-H stretch aliphatic), 1345 (in-plane $\mathrm{O}-\mathrm{H}$ bend) and $1111 \mathrm{~cm}^{-1}$ (C-O stretch), which were consistent in all binary systems with the drug. This indicates the absence of drug-excipient interactions because all specific peaks of the drug were present in the solid dispersion.

\section{IDR studies}

Figure 6 shows the amount of pure FEN dissolved from the obtained solid dispersions in a $0.5 \%$ SLS solution at a time 


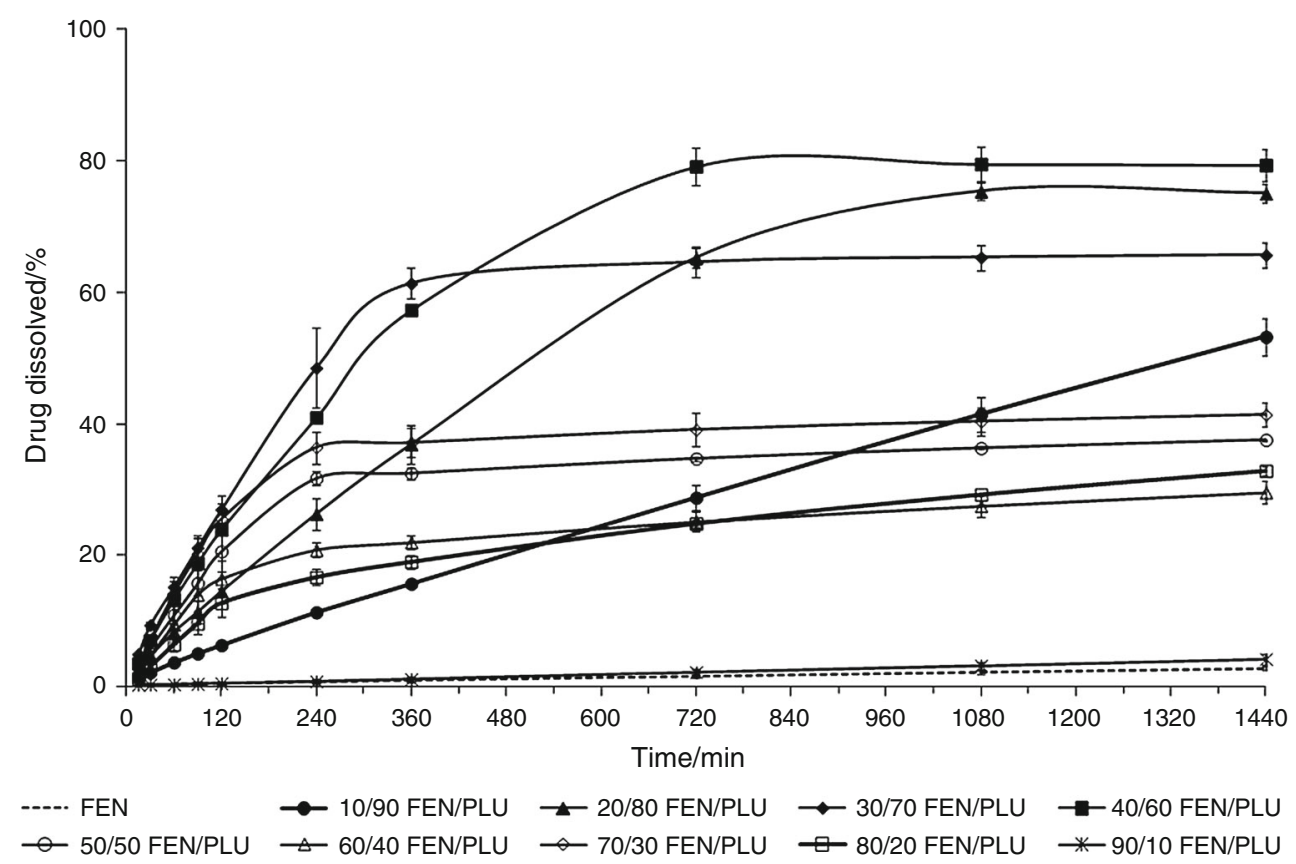

Fig. 7 Dissolution profiles of FEN and its dispersions with PLU within $24 \mathrm{~h}$

of $120 \mathrm{~min}$. The IDRs of various solid dispersions were measured and compared with that of pure FEN. Kaplan noted that compounds with an IDR below $0.1 \mathrm{mg} \mathrm{cm}^{-2} \mathrm{~min}^{-1}$ typically exhibited a dissolution rate-limited absorption [22]. The IDR of pure FEN of $0.003 \mathrm{mg} \mathrm{cm}^{-2} \mathrm{~min}^{-1}$ falls into this category. The IDR of FEN, its solid dispersions with PLU, and the linear relationship between the amount of the dissolved drug and time are provided in Table 2 . The results suggest that the dissolution rate of FEN in solid dispersions is higher than that of the pure substance. After $120 \mathrm{~min}$ of testing, depending on the formulation composition, the IDR increased by 1.1-fold for the 90/10 FEN/PLU composition and by more than 100-fold for the 30/70, 40/60, and 70/30 FEN/PLU \%w/w formulations. These results correspond to a dissolved amount of 27,24 , and $25 \%$ of the total active pharmaceutical ingredient (API) for the 30/70, 40/60, and 70/30 FEN/PLU \%w/w formulations, respectively. In addition, only $0.48 \%$ of the pure FEN was dissolved. As the polymer concentration increases, the dissolution increases due to the enhanced disaggregation and wetting of FEN particles in the presence of hydrophilic groups of the polymer and surface active properties of PLU. Figure 7 shows the dissolution profiles of FEN from solid dispersions within $24 \mathrm{~h}$. A dissolution profile plateau was observed after $1440 \mathrm{~min}$ of dissolution tests for solid dispersions containing 30/70, 40/60, and 70/30 \% w/w of FEN/PLU. This plateau corresponds to the dissolved drug percentage, specifically, 66,79 , and $41 \%$, respectively.

\section{Conclusions}

In the present study, PLU was employed to prepare solid dispersions because it acts as a hydrophilic carrier and enhances the dissolution rate of the drug. In the dissolution medium, the hydrophilic polymer undergoes a rapid dissolution and attains a high concentration in the diffusion layer of the drug. Thus, this improves the drug dissolution in this layer and increases the dissolution rate of the substance. The dissolution rate of FEN from the obtained solid dispersions was higher than that of the pure drug. The DSC studies revealed that FEN and PLU formed a simple eutectic system that contained $16.5 \% \mathrm{w} / \mathrm{w}$ of FEN at the eutectic point. FTIR spectra, DSC, and XRPD studies indicated the absence of drug-polymer interactions.

Open Access This article is distributed under the terms of the Creative Commons Attribution 4.0 International License (http://crea tivecommons.org/licenses/by/4.0/), which permits unrestricted use, distribution, and reproduction in any medium, provided you give appropriate credit to the original author(s) and the source, provide a link to the Creative Commons license, and indicate if changes were made. 


\section{References}

1. Granero GE, Ramachandran C, Amidon GL. Dissolution and solubility behavior of fenofibrate in sodium lauryl sulfate solutions. Drug Dev Ind Pharm. 2005;31:917-22.

2. Brunton LL, Lazo JS, Parker KL, editors. Goodman and Gilman's the pharmacological basis of therapeutics. 11th ed. New York: McGraw-Hill Companies; 2006.

3. Law D, Wang W, Schmitt EA, Qiu Y, Krill SL, Fort JJ. Properties of rapidly dissolving eutectic mixtures of poly(ethylene glycol) and fenofibrate: the eutectic microstructure. J Pharm Sci. 2003;92:505-15.

4. He H, Yang R, Tang X. In vitro and in vivo evaluation of fenofibrate solid dispersion prepared by hot-melt extrusion. Drug Dev Ind Pharm. 2010;36:681-7.

5. Kataria MK, Bhandari A. Solubility and dissolution enhancement: technologies and research emerged. J Biol Sci Opin. 2013;1:105-16.

6. Vogt M, Kunath K, Dressman JB. Dissolution enhancement of fenofibrate by micronization, cogrinding and spray-drying: comparison with commercial preparations. Eur J Pharm Biopharm. 2008;68:283-8.

7. Bhise SD. Ternary solid dispersions of fenofibrate with poloxamer 188 and TPGS for enhancement of solubility and bioavailability. Int J Res Pharm Biomed Sci. 2011;2:583-95.

8. Yousaf AM, Kim DW, Kim JO, Chang PS, Baek HH, Lim SJ, Cho KH, Yong CS, Choi HG. Characterization of physicochemical properties of spray-dried solid dispersions loaded with unmodified crystalline fenofibrate. Curr Pharm Anal. 2015;11: $139-44$

9. Babu AM, Rao BP, Sudhakar P. Development and characterization of novel self-microemulsion drug delivery system of low solubility drug "fenofibrate" for improved oral bioavailability. Int J Biol Pharm Res. 2012;3:616-23.

10. Ajmera A, Deshpande S, Kharadi S, Rathod K, Patel K, Patel P. Dissolution rate enhancement of atorvastatin, fenofibrate and ezetimibe by inclusion complex with $\beta$-cyclodextrin. Asian $\mathbf{J}$ Pharm Clin Res. 2012;4:73-6.
11. Jamzad S, Fassihi R. Role of Surfactant and $\mathrm{pH}$ on dissolution properties of fenofibrate and glipizide - a technical note. AAPS Pharm Sci Tech. 2006;7:E33.

12. Yadav VB, Yadav AV. Enhancement of solubility and dissolution rate of BCS class II pharmaceuticals by nonaquious granulation technique. Int J Pharm Res Dev. 2010;1:1-8.

13. Yadav VB, Yadav AV. Enhancement of solubility and dissolution rate of fenofibrate by melt granulation technique. Int $\mathrm{J}$ Pharm Tech Res. 2009;1:256-63.

14. Kalidova A, Fischbach M, Kleinebudde P. Application of mixtures of polymeric carriers for dissolution enhancement of fenofibrate using hot-melt extrusion. Int J Pharm. 2012;429: $58-68$.

15. Bonthagarala B, Nama S, Nuthakki S, Kiran KV, Pasumarthi P. Enhancement of dissolution rate of fenofibrate by using various solid dispersion techniques. World J Pharm Pharm Sci. 2014;3:914-32.

16. Swati S, Mohan MV. Formulation, characterization, in vitro and in vivo evaluation of fenofibrate solid dispersions. Adv Sci Eng Med. 2014;6:625-36.

17. Tejas P, Patel LD, Tamir P, Sunil M, Tushar P. Enhancement of dissolution of fenofibrate by solid dispersion technique. Int J Res Pharm Sci. 2010;2:127-32.

18. Tejas P, Patel LD, Adeshara SP, Timir P, Sunil M, Tushar P. Dissolution enhancement of fenofibrate by solid dispersion technique. Curr Pharm Res. 2011;1:127-34.

19. Islam MdS, Mehjabeen K, Mustary I, Khan F, Jalil R. Improvementof dissolution of ibuprofen and fenofibrate by poloxamer based solid dispersion. Bangladesh Pharm J. 2010;13:54-9.

20. Irwan AW, Berania JE, Liu X. A comparative study on the effects of amphiphilic and hydrophilic polymers on the release profiles of poorly water-soluble drug. Pharm Dev Technol. 2014. doi:10. 3109/10837450.2014.991877.

21. Rycerz L. Practical remarks concerning phase diagrams determination on the basis of differential scanning calorimetry measurements. J Therm Anal Calorim. 2013;113:231-8.

22. Kaplan SA. Biopharmaceutical considerations in drug formation design and evaluation. Drug Metab Rev. 1972;1:15-34. 